\title{
Tools and Technology for Effective Remote Work
}

\author{
Balu N. Ilag \\ UC \& Collaboration Expert \\ Juniper Networks
}

\begin{abstract}
Office work refers to work that is generally done in an office building designated explicitly for administrative and professional duties. Remote work relates to business operations and professional tasks outside of the office space like telecommunication or working from home. Many companies believe that office spaces are more productive because the collective energy of having other people around who are working towards the same goal promotes innovation, collaboration, and collision. Production is at a faster pace when there are a laser focus and no distractions. More impromptu and immediate interactions and consultations create a deep cultural connection and assistance on tasks that one cannot complete independently (Haynes et al., 2017).

The office space is not without its challenges. Productivity can be limited due to distractions that one is unlikely to encounter when working remotely, such as noise and commotion, interruptions from other workers dropping into one's office space, impromptu meetings, and gossip sessions (Haapakangas et al., 2018). Commutes to and from the office building result in time wastage and fatigue. Skipping a commute has the impact of letting one customize the workday and work longer hours due to the reduced stress levels (Lin \& Bao, 2019).
\end{abstract}

\section{General Terms}

Remote work, Effective Work from Home, Tools for Remote work, Effective Remote work using Tools, Effective Tools for remote work.

\section{Keywords}

Remote work, Effective Remote work, Tools and Technology for Remote work, VoIP, Data Network

\section{INTRODUCTION}

Office work refers to work that is generally done in an office building designated explicitly for administrative and professional duties. Remote work relates to business operations and professional tasks outside of the office space like telecommunication or working from home. Many companies believe that office spaces are more productive because the collective energy of having other people around who are working towards the same goal promotes innovation, collaboration, and collision. Production is at a faster pace when there are a laser focus and no distractions. More impromptu and immediate interactions and consultations create a deep cultural connection and assistance on tasks that one cannot complete independently (Haynes et al., 2017).

The office space is not without its challenges. Productivity can be limited due to distractions that one is unlikely to encounter when working remotely, such as noise and commotion, interruptions from other workers dropping into one's office space, impromptu meetings, and gossip sessions (Haapakangas et al., 2018). Commutes to and from the office building result in time wastage and fatigue. Skipping a commute has the impact of letting one customize the workday and work longer hours due to the reduced stress levels (Lin \& Bao, 2019).

\section{WORKING STYLE CHANGE FROM OFFICE TO REMOTE WORK}

Employees are advocating for working remotely, and many organizations are working towards the policy of remote work. The flexibility awarded by remote work is desirable, especially to millennial employees. They still want to spend time on their hobbies and talents and dedicate time to their families. Employers enjoy the same perks, with the added advantage of lesser costs on in-house office facilities and rent on large office spaces (Hunter, 2019). Bloom and Lang's experiment on increased productivity (2018) revealed that remote workers were more productive than office workers by $13 \%$ and worked $9.5 \%$ longer.

Similarly, remote work offers an opportunity to workers who are geographically further away from the firm and allows employers to recruit the top talent without limiting workers to only those who can physically make it to the office. Variety creates diversity and culture, which helps develop dynamics for problem-solving due to the varying experiences, expertise, and viewpoints that people from different works of life provide. It is easier to appeal to a broader range of consumers if they are well-represented in an organization (Rintel et al., 2020).

While others are more productive when working remotely, some workers find it hard to be productive when working in isolation (Bell, 2020). Great geographical distances and time differences can be disadvantageous because simple queries may take hours to be sorted if it is not office hours in the other time zone. Sometimes, workers may be forced to work late into the night to accommodate the clients' time zones. Discipline might be hard to establish when establishing working hours and a routine (Elshaiekh et al., 2018). Just as in-house offices require equipment, remote working might require technological and communication equipment that might be hard to come by or be region-dependent. The resources needed for sufficient remote work are meant to counter the challenges that remote workers face when accomplishing their tasks. Therefore, to get the best out of remote working, organizations have to develop plans that can implement intelligent business software and tools and increase value on the influence of business communication and collaboration tools (Willcocks, 2020).

\section{COLLABORATION AND COMMUNICATION}

Communication and collaboration require a combination of intrapersonal, communication, and problem-solving skills that are sustainable for a long-term basis. Excellent communication is the basis of trust and is achieved by listening intently, offering encouragement and support, and talking with respect for the information (Rochester, 2017). 
Intrapersonal skills involve maintaining relationships, building new ones through proper communication, appreciating others' efforts, solving problems, and disagreements amicably and being considerate. Problemsolving skills refer to analyzing a situation correctly and developing the best possible solution (Banaeianjahromi \& Smolander, 2019).

Collaboration differs from teamwork because it involves working with people on different levels and sectors with differing sets of skills, providing a more comprehensive range of options and solutions. However, differing skill sets suggest different thought processes, modes of operation, and communication, making it hard to establish a united team. As such, collaboration can only be created through proper communication, cooperation, coordination, and teamwork. Remote working faces teamwork and collaboration threats due to its nature of remote operations (Scott \& Queens, 2017). Some lack the appropriate communication tools to sustain the standards of service. Those who have underlying software and communication tools require the right systems to utilize them for maximum benefit. Therefore, as companies move towards remote work, it is essential to establish the right balance of software and tools with working systems and management (Rebentisch \& Prusak, 2017).

Communication breakdown is fatal for the running of any business. The lack of serial communication plagues remote employees and teams. Emails are not enough. Tools to expedite real-time communication are valuable firstly because they reduce the feeling of isolation and improve the perception of being a member of a team (Sadgrove, 2016). Regularly interacting with the people, one works with raises morale, helps build trust and engagement, and reduces competition. The workers focus on working alongside each other to achieve their goals. Constant and timely feedback addresses any mistakes that the remote team is making before much damage is done. It keeps all the members on their toes because supervisors can track how both short-term and longterm goals are being achieved. Likewise, excellent performance can be praised to motivate the employees to keep doing good work. Collaboration is hindered by the lack of real-time communication (Erlandia, 2016).

\section{COLLABORATION TECHNOLOGY AND MICROSOFT TEAMS}

The communication and collaboration problems in remote working cause productivity gaps and inconsistencies. The lack of proper schedules disrupts routines, disables workflow, and creates bad habits that lead to a failure to accomplish goals and objectives set. Productivity gaps can destroy hours of progress without proper systems for time and project management. Therefore, when selecting a technological system to it is crucial to choose one that caters to most, if not all, of the difficulties that a remote team is facing. Communication and information-sharing should be smooth so that everyone can participate in creative brainstorms, feedback loops, and cross-functional decision-making for effective collaboration (Deshpande et al., 2016).

\subsection{Unified Communications and Collaboration (UCC) Technology}

Unified Communications and Collaboration (UCC) technologies are software that enables enterprise communication in real-time and asynchronous cooperation proficiency. It uses a single interface comprising collaboration solutions and Individual Unified Communications (IUC) methods to improve productivity through connectivity. During these times, with a worldwide pandemic, almost all companies and organizations have been forced to work remotely to minimize contact and infection (Silic et al., 2017). One essential UCC tool that has proven useful and effective for most organizations is Microsoft Teams.

Microsoft Teams is an all-in-one collaboration application that helps teams working remotely to have conversations and stay organized by allowing firms to create their inside channels for on-the-spot, real-time meetings, chat conversations, and file-sharing. Microsoft teams can set up audio and video calls even with people who do not use the Teams software. It can hold, organize, and schedule meetings through the Outlook Calendar. Activity is meant for live interactions among the Team members through messages, conversations, mentions, replies, and more (Ilag, 2018).

The most significant advantage for Microsoft Teams is that it provides most of the necessary communication and collaboration features for free as long as one has an active subscription to Microsoft Office 365. The free version offers unlimited video and audio calls, messaging, and activity with ten gigabytes storage for Team data and two gigabytes for personal data (Ilag, 2019). It thus lowers organizational expenditure. Another great advantage is that Microsoft Teams seamlessly integrates with Office 365 applications like Excel, Word, Access, PowerPoint, Project, and OneNote. One can use Skype, Exchange, SharePoint, and Yammer with Microsoft Teams (Hubbard \& Bailey, 2018).

Microsoft Teams addresses the problem of isolation that remote and dispersed teams face by including the Chat option. Teams can hold project meetings and keep up-to-date with the relevant information concerning progress and accomplishments by creating a group chat. The Group Chat feature allows the group to send links, documents, images, and any necessary information, allowing individual remote teams to have all their data in one place to avoid project mixup. It will enable one-on-one conversations for regular consultations and data transfer that do not require the entire team (Ilag, 2020). As such, data is transferred flawlessly, and anyone who is part of the team can access it without wasting time going through contact channels. It provides organizational privacy since one-on-one chats are private to the participants only while group chats are visible to all group members. Everyone is made to feel important as part of the team since Microsoft Teams chat does not develop a superiority hierarchy.

Every project, department, sector, or client data and conversation in Microsoft Teams can be stored separately for organization and easy access. For every user, Microsoft team allows every user to create up to two hundred and fifty teams and be a member of up to one thousand teams. Up to ten thousand people can be members of one team, and the limit for organization-wide teams is five thousand members. The teams' difference is that private teams require administration permission to join and are mostly protected by passcodes. Public teams are free for anyone in the company to join, and organization-wide teams automatically add everyone in the organization (Hubbard \& Bailey, 2018).

The global administrators in organizational teams have the advantage of automatically adding everyone who is a member of the firm into the team. As new members join, Active Directory updates them into the organization and eaves no one out. Unlicensed users within the organization are added into 
the group and assigned an Exploratory License, which means automatic additions that are yet to be signed in by the Team owners or administration are allowed to use the Microsoft Team features on a trial basis (Hubbard \& Bailey, 2018). People who leave the team are automatically disabled from the organization-wide team, a significant security feature that prevents data trading from previous employees. It allows employers and managers to virtually keep track of their remote employees since members cannot leave the organization-wide team unless the administrator removes them. Thus, it establishes an accountability system and encourages all workers to create a schedule and stick to it (Ilag, 2020).

Organization-wide teams provide a general channel where all members can monitor the activities going on. Microsoft recommends allowing only the team owners to post the required announcements on the general channel to reduce an influx of unnecessary information that overshadows the crucial information. All the teams are given a limit of two hundred channels. In case the team owners delete any channels, they are counted within the two-hundred channel limit to provide a chance to restore the channel but is permanently deleted after the month expires and is no longer counted within the channel limit. Consequently, team owners have the privilege of setting channel priority ensures that the most important channels are auto displayed to steer discussions and engagement towards specific topics and directions (Hubbard \& Bailey, 2018).

Video calls and conferences on Microsoft Teams are scheduled using the Outlook Calendar, which comes with an automatic reminder after accepting the invitation to join the meeting that ensures everyone attends the meetings (Hubbard \& Bailey, 2018). Scheduling the meetings allows remote teams to account for time differences by selecting a meeting time that does not inconvenience others. New members of remote teams find it easier to integrate into the team if they can interact with their new workmates. Necessary recruitment process like orientations has become easier since interactive ones are more effective than brochures and written documents (Cooper, 2020).

Given that eighty percent of all communication is derived from visual cues, remote team meetings have been made more accessible since Microsoft Teams allows up to twenty people to join in one video chat at a time and up to one hundred participants. The extra people above the twenty limit are usually more of an audience than participants since the video feature does not work for them. PowerPoint presentations can be made through Microsoft Teams video conference calls with a limit of two gigabytes (Hubbard \& Bailey, 2018). Next to in-person meetings, video conferencing is the only meeting that provides all the required communication cues from visual, tonal, audio, facial expressions, and postures. It encourages engagement and minimizes communication errors. It gives a more accurate gauging of decision-making because the reception of the decisions is visible in real-time. The entire decision-making process is made time-conscious since feedback is immediate (Ilag, 2020).

Microsoft Teams stores meeting details, minutes, and documents shared during the video chat for up to twenty days if one needs to clarify or remind themselves of any data they may have forgotten or overlooked. Afterward, Teams can upload call data to Microsoft Stream, where it is available anytime and anywhere for local download to one's local drive. The Microsoft Stream feature is useful because of the ability to store all that was said, and all the documents exchanged without losing any data to translation or a low attention span. The team members can go back and watch the meeting or discussion repeatedly (Hubbard \& Bailey, 2018).

The collaboration and seamless communication that Microsoft Teams has been able to provide for the teams and firms that use it have been useful in improving overall productivity. Organizational collaboration is five times more likely to lead to higher performance (Silic \& Sammer, 2017). Microsoft Teams' lowered costs, improved productivity, and shortened timelines have raised return on investments (ROI). A survey showed that companies using Microsoft Teams had reduced their email volume by $48 \%$, reduced their physical meetings by $25 \%$, and increased their overall productivity by $32 \%$ (Ilag, 2018).

The different channels in Microsoft Teams are used by various departments to easily communicate and share information because they belong to the same team. As such, Teams break down depositories and allow inter-departmental communication for faster communication and easier collaboration that saves time and minimizes friction. Streamlined workflows create an able company that costefficiently replies to market demands. The business can dedicate more time to customer development and fulfillment and organizational expansion (Hafermalz \& Riemer, 2016). Connecting the customers or clients to the right team member that can offer them direct service and assistance reduces the resources and time that is usually involved in customer care services without having to compromise on quality. Minimizing the use and reliance on email services gets rid of version control problems from delays by sharing up-to-date data in real-time (Begel, 2019).

\section{CONCLUSION}

The overall advantage of using the right Unified Communication \& Collaboration tool like Microsoft Teams for remote work is that it bridges the physical and virtual communication and collaboration gaps, making it possible to enjoy the perks of remote work without most of the challenges and disadvantages. As we move towards a working environment, it is important not to have to compromise on the quality of work. Microsoft Teams can save time and bridge distance without having to compromise on the quality or quantity of work output and customer satisfaction while still saving on costs. They also have the added advantage of cloud storage of data, automatic workflow and updates, event planning and scheduling, and security through end-to-end encryption.

\section{ACKNOWLEDGMENTS}

Our thanks to the experts who have contributed towards this paper and researcher who has contributed for UCC technology and Tools. Special thanks to our family member for their continues support.

\section{REFERENCES}

[1] Banaeianjahromi, N., \& Smolander, K. (2019). Lack of communication and collaboration in enterprise architecture development. Information Systems Frontiers, 21(4), 877-908.

[2] Begel, A. (2019, May). Best practices for engineering AI-infused applications: lessons learned from Microsoft teams. In 2019 IEEE/ACM Joint 7th International Workshop on Conducting Empirical Studies in Industry (CESI) and 6th International Workshop on Software Engineering Research and Industrial Practice (SER\&IP) 
(pp. 1-1). IEEE.

[3] Bell, C. J. (2020). Feeling remote: Factors influencing isolation in remote workers.

[4] Bloom, N. (2018). To raise productivity. Let More Employees Work from Home, 27.

[5] Cooper, D. (2020). The digital mediator: How technology is setting the new standard. Proctor, 40(5), 24-27.

[6] Deshpande, A., Sharp, H., Barroca, L., \& Gregory, P. (2016). Remote working and collaboration in agile teams.

[7] Elshaiekh, N. E. M., Hassan, Y. A. A., \& Abdallah, A. A. A. (2018, November). The impacts of remote working on workers performance. In 2018 International Arab Conference on Information Technology (ACIT) (pp. 15). IEEE.

[8] Erlandia, D. R. (2016, January). Event management, a flourishing business in creative industries theme: Communication among creative industries. In International Conference on Transformation in Communication (ICOTIC).

[9] Haapakangas, A., Hallman, D. M., Mathiassen, S. E., \& Jahncke, H. (2018). Self-rated productivity and employee well-being in activity-based offices: The role of environmental perceptions and workspace use. Building and Environment, 145, 115-124.

[10] Hafermalz, E., \& Riemer, K. (2016). The work of belonging through technology in remote work: A case study in telenursing.

[11] Haynes, B., Suckley, L., \& Nunnington, N. (2017). Workplace productivity and office type. Journal of Corporate Real Estate.

[12] Hubbard, M., \& Bailey, M. J. (2018). Mastering Microsoft Teams. End-User Guide to Practical Usage,
Collaboration, and Governance. Berkeley: Apress.

[13] Hunter, P. (2019). Remote working in research: An increasing usage of flexible work arrangements can improve productivity and creativity. EMBO Reports 20(1), e47435.

[14] Ilag, B. N. (2018). Introduction: Microsoft Teams. In Introducing Microsoft Teams (pp. 1-42). Apress.

[15] Ilag, B. N. (2020). Managing and controlling Microsoft Teams. In Understanding Microsoft Teams Administration (pp. 37-229). Apress.

[16] Lin, J. H., \& Bao, S. (2019). The effect of sit-stand schedules on office work productivity: A pilot study. Work, 64(3), 563-568.

[17] Rebentisch, E., \& Prusak, L. (2017). Integrating program management and systems engineering: Methods, tools, and organizational systems for improving performance. John Wiley \& Sons.

[18] Rintel, S., Iqbal, S., Suh, J., Czerwinski, M., Mark, G., \& Teevan, J. (2020). Remote work and well-being.

[19] Rochester, M. R. (2017). Synchronous communication and its effects on the collaboration of professional workplace employees engaged in a problem activity.

[20] Sadgrove, K. (2016). The complete guide to business risk management. Routledge.

[21] Scott, B. B., \& Queen's, I. R. C. (2017). Creating a collaborative workplace: Amplifying teamwork in your organization.

[22] Silic, M., Back, A., \& Sammer, T. (2017). Employee acceptance and use of unified communications and collaboration in a cross-cultural environment. In Remote Work and Collaboration: Breakthroughs in Research and Practice (pp. 1-22). IGI Global.

[23] Willcocks, L. P. (2020). Remote working: Here to stay? LSE Business Review. 